\title{
A Proposed Framework for Photos Copyright Protection in Facebook
}

\author{
Mohammad Alaa Hussain Al-Hamami, PhD \\ Management Information \\ Systems Department \\ Applied Science University, \\ Kingdom of Bahrain
}

\begin{abstract}
Because of the huge number of users, Facebook becomes the largest social network on earth. Although, Facebook is continuously developing its features but doesn't provide yet a suitable mechanism to protect the content copyright in an automatic way.

This paper suggests a framework proposal to protect the copyright of photos in Facebook. This will be done by adding a watermark to photo that will be uploaded. The watermark contains the name of the profile that uploaded photo and date of posting it. This profile will be considered as the owner of the photo on Facebook. If the uploaded photo already contains a Facebook watermark, the profile that already owned the photo will be notified by Facebook that there is another profile trying to upload the same photo. The original owner will decide to give the permission for the photo to be posted again on Facebook or not. If the photo's owner allows the photo to be posted the watermark information will be displayed in the post. The system doesn't allow the new user to put his/her water mark on the photo. In this way, even if a profile posted a watermarked photo it will be clear to everyone who is the original owner and when the profile posted it.
\end{abstract}

\section{General Terms}

Security, Information Hiding, Image Watermark, Copyright, Social Networks

\section{Keywords}

Digital Watermark, Photo Copyright, Facebook Watermark, Photo Owner, Proposed Framework.

\section{INTRODUCTION}

Because of its popularity, Facebook as a social network is the largest country on earth due to the huge number of its users. Worldwide, there are over 1.79 billion monthly active Facebook users which is a 16 percent increase year over year. Five new profiles are created every second. Photo uploads total 300 million per day. Every 60 seconds on Facebook: 510,000 comments are posted, 293,000 statuses are updated, and 136,000 photos are uploaded. 4.75 billion pieces of content shared daily as of May 2013, which is a 94 percent increase from August 2012 [1]. 97\% of the Interbrand Top 100 Global brands have dedicated Facebook accounts, and 93\% posted content in the month of May 2016 [2].

From the previous numbers, it is clear that Facebook became the main social network for business and individuals. Although, Facebook keeps continuously updating its features in order to remain in the forefront, but protecting the content copyright is one of the biggest issues for Facebook users. Facebook relies on reporting by users to protect the copyright of content. With the enormous amount of content, Facebook need an automated process to protect the copyright. One of the best ways to protect copyrights is by using watermarks.

\section{DIGITAL IMAGE WATERMARK CONCEPTS}

With the tremendous usage of the Internet and its applications and with the massive amount of digital content that is posted every day, digital watermark becomes one of the most important topic in the field of information security. Digital watermark is a part of information hiding and it considered one of the effective means of digital products copyright disputes.

Research on digital watermark for copyright protection has a great significance. If the digital image watermark was robust, it will have a great practical value in the copyright protection of digital image. For that reason, digital watermarks can be useful in protecting the copyright of digital products, and that will have a very broad commercial value [3].

Digital watermark in images is the process by which a discrete data stream is hidden within an image imposing imperceptible change in the image. It can be classified into different categories, the first category depends on the type of the domain in which the data embedding take place, the domains can be spatial or transform. The second category is the resistance of watermark against attack; fragile watermarks will be destroyed by image processing methods, and the change in the watermark is easy to be detected, this will give information about image completeness; robust watermarks are robust against most of image processing methods, so the watermark can be extracted from a strong attacked watermarked image. The third category depends on the type of information needed for retrieving watermark in the extraction process [4].

The following are the requirements for a successful digital image watermark [5]:

- Imperceptibility is defined as the similarity between the original image and the watermarked image.

- Robustness means a digital image watermark should survive against various distortions or attacks.

- Fragility is the ability to detect an alteration of the watermark due to tampering attempts.

- Information rate means the amount of information for a digital watermark that achieve the appropriate rates for a given distortion constraint and a set of attacks. 


\section{COPYRIGHT IN FACEBOOK}

Facebook generally protects the copyright content by allowing the user to report any issue related to copyright.

Facebook wrote in its copyright section "the FAQs will provide some information about copyrights, including how you can protect your own copyrighted works and avoid infringing the copyrights of other people when posting to Facebook. As well as how Facebook addresses reports of copyright infringement. If you believe that someone is using your copyrighted work without your permission, you can fill out the Copyright Report Form [6] to report the incident".

Figure 1 shows Facebook reporting form:

\section{Copyright Report Form}

Use this form to report content which you believe violates or infringes your copyright.

To be sure you're in the right place, what best describes your issue?

I lost access to a group or Page I used to manage

Ifound a fake account that is pretending to be me

I found inappropriate or abusive content

I am being bullied or harassed

I found content which I believe violates my copyright

I found content which I believe violates my trademark

I appear in content that I do not want displayed

My child (or someone I am legally responsible for) appears in content that I do not want displayed

My account has been hacked

My name (or the name of my organization) is being used without my permission

Fig 1: Facebook Reporting Form [6]

Facebook also mentioned that laws in different countries may vary, and if you need more information on copyright law, you can visit the website of the U.S. Copyright Office or the World Intellectual Property Organization (WIPO). And they made it clear that Facebook can't provide legal advices.

About reporting a copyright infringement, Facebook clarifies it's a manual process and it must be done by the copyright owner:

"If you believe someone is infringing your copyright, you can report it to us by filling out this form. You can also contact our designated agent. If you contact our designated agent, please be sure to include a complete copyright claim in your report.

Before you submit a report of copyright infringement, you may want to send a message to the person who posted the content. You may be able to resolve the issue without contacting Facebook.

Remember, only the copyright owner or their authorized representative may file a report of copyright infringement. If you believe something on Facebook infringes someone else's copyright, you may want to let the rights owner know" [8].

So, it is very clear from the previous that although Facebook is keeping developing its features, but the copyright protection process still need more improvement. The copyright owner must discover the copyright infringement and reported to Facebook, and this is very difficult to implement because of:

(1) The huge amount of content that is published every day by users.

(2) The nature of Facebook that depends on connecting friends with friends, so if the content that had been posted wasn't public there is a big chance that the copyright owner will not know about it.

\section{THE PROPOSED FRAMEWORK}

The aim of the proposed framework is to protect photos copyright in Facebook; this will be done by embedding an invisible Facebook watermark to any photo that will be uploaded and doesn't contain a Facebook watermark. The watermark will include the following information:

1. The profile name that will upload the photo (the profile will be considered as the owner of the photo.)

2. The date when the photo will be posted.

For example, if profile $X$ uploaded a photo on $1 / 1 / 2017$ the watermark will be (Posted by: X, 1/1/2017)

Figure 2 illustrates posting a photo for the first time on Facebook by profile $\mathrm{X}$ : 


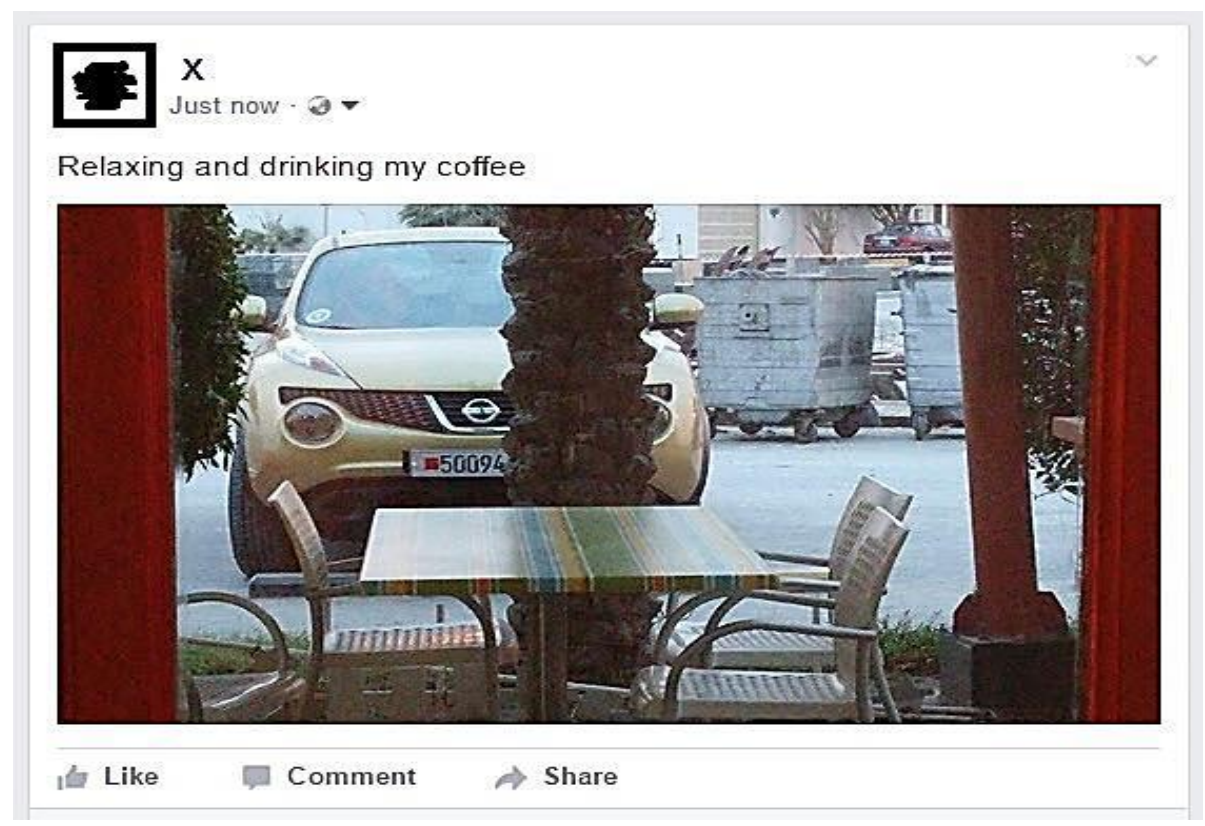

Fig 2: The photo is uploaded for the first time by profile $X$

If the uploaded photo already contains a Facebook watermark, the owner of the photo who had published the original photo on Facebook will be notified that there is another profile trying to upload the same photo. The owner will decide to allow the photo to be posted or not. If the owner allowed the photo to be posted, it will be posted by the other profile and the owner watermark information will be displayed with the post. Figure 3 illustrates that, there is another profile trying to upload the same photo that been posted by profile $\mathrm{X}$ on $1 / 1 / 2017$. If profile $X$ allows the photo to be posted by the other profile, the watermark information will display with the post:

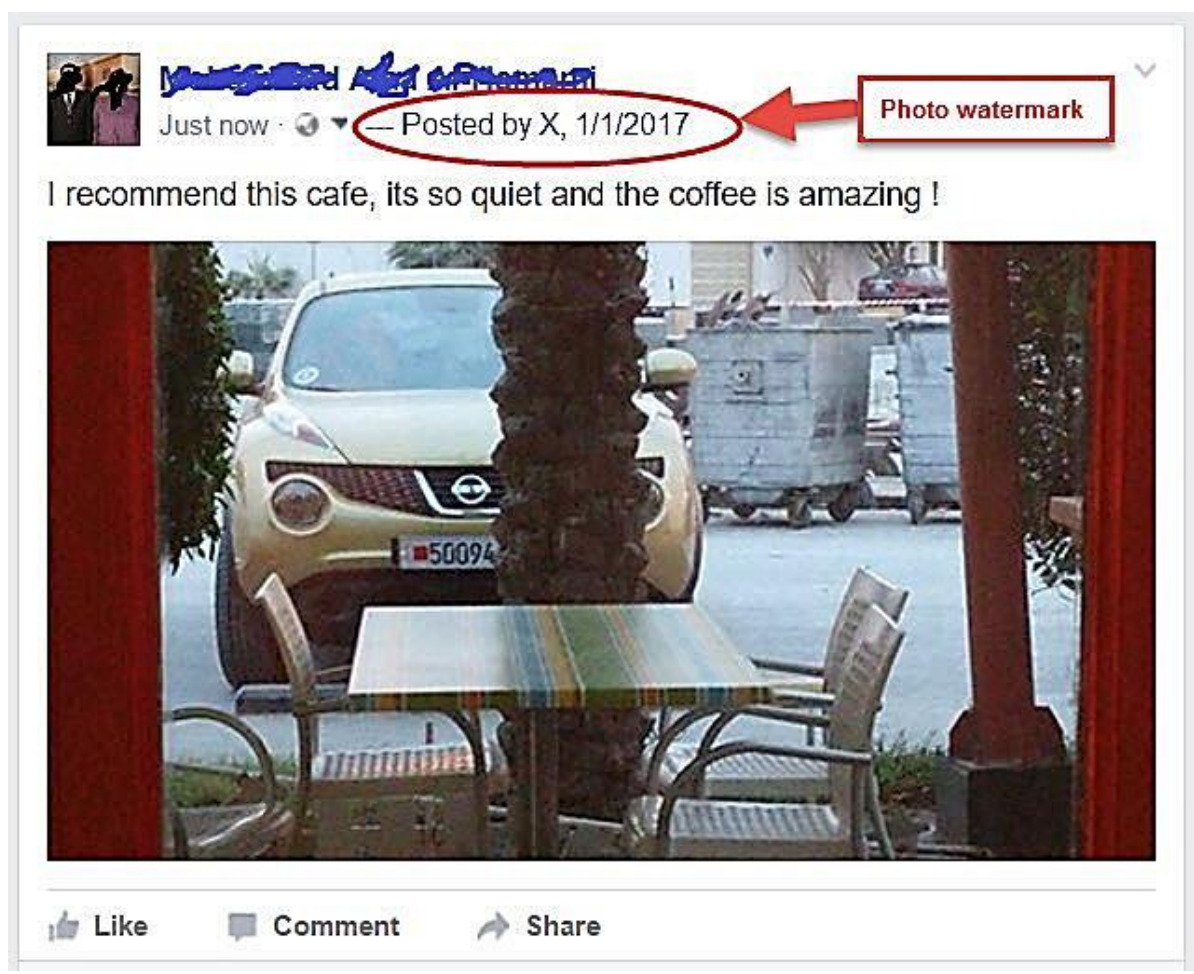

Fig 3: Another profile posted the same photo that been post by profile $X$ on $1 / 1 / 2017$

There are many information hiding tools that can be used successfully to hide information in Facebook photos like Stegobot and Secretbook [9]. These tools can embed watermarks to photos and extract them later if there is a need for that.

\subsection{Framework Flow Diagram}

Figure 4 clarify the flow diagram of the proposed framework for photos copyright protection in Facebook: 


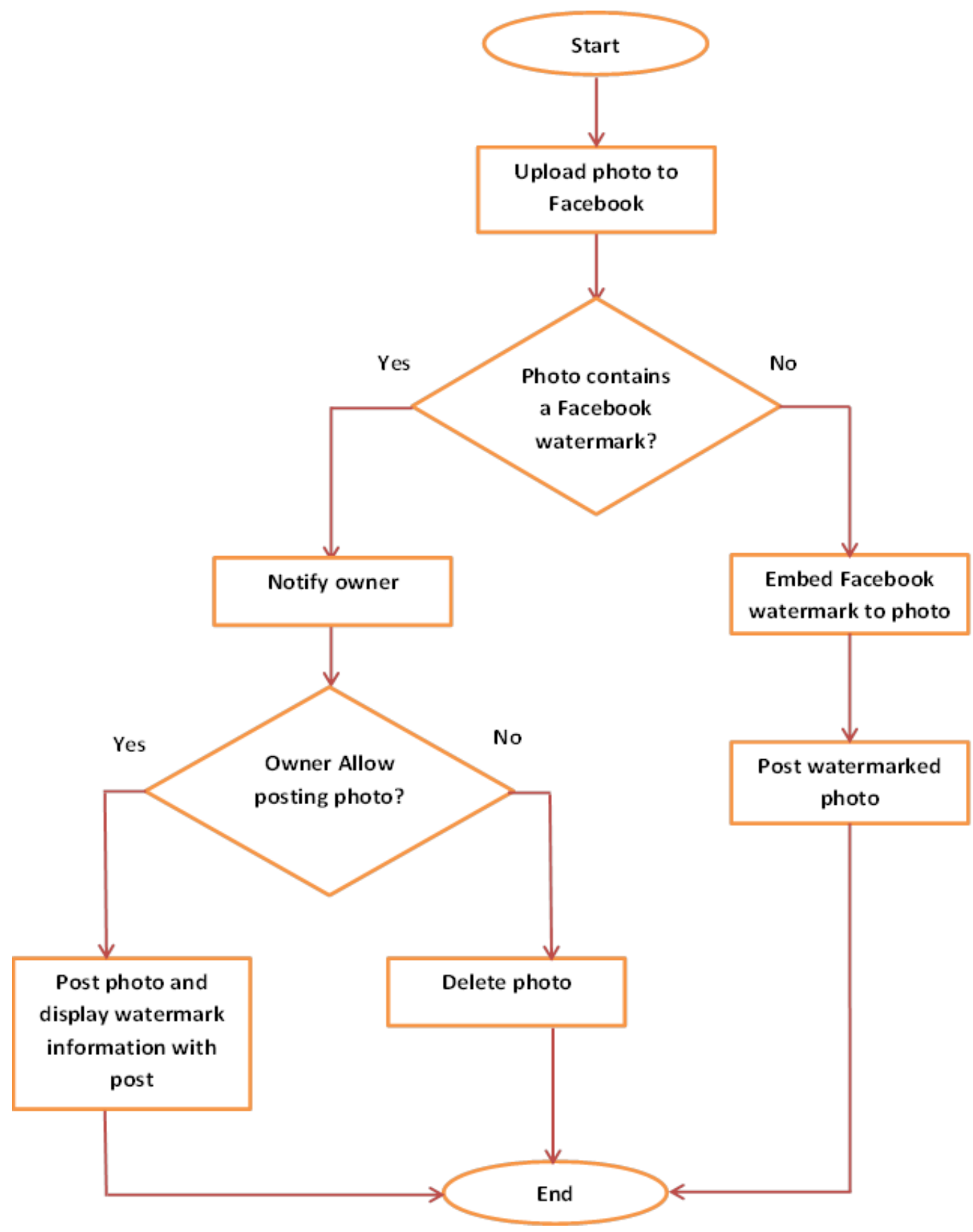

Fig 4: Framework Flow Diagram

\subsection{The Framework Steps}

The framework will consist of the following steps:

Input: Photo to be uploaded

Output: Decide to post a Facebook watermarked photo or not

Step1: Upload photo

Step2: Check photo if it contains a Facebook watermark

If photo contain Facebook watermark

Notify owner

If owner allow photo to be posted with the post
Post photo and display watermark information
Else

Delete photo

Else

Embed Facebook watermark to photo

Post Photo

Step3: End

\section{CONCLUSIONS}

The proposed framework will protect the copyright of photos that will be posted on Facebook. This will be done by embedding a suggested invisible Facebook watermark in any photo that will be posted on Facebook. If a Facebook profile is trying to post a photo that already have a Facebook watermark, the original owner of the photo (the profile who 
published the photo first on Facebook) will be notified that there is another profile trying to post the same photo on Facebook without sharing it by using the regular sharing process, the profile who own the photo will decide to allow the photo to be posted or not; in this way, every photo on Facebook will contain a watermark that describe which profile is the owner of the photo and when the photo has been posted. The suggested framework is necessary for Facebook because of the following reasons:

1. Facebook profiles will be responsible about the photos that they will post; this will enhance the quality of the content because profiles owners will think twice before posting any an appropriate photo.

2. The source of the original photo (the profile who posts the photo first), and the date of posting will be always known by extracting the watermark information.

3. It will be easy to prosecute judicially any profile posted a photo without having the right to post it because the watermark contains the needed information for that.

4. The profile who owned the photo will be notified if there is another profile trying to post the same photo; this is a critical point because without using the suggested framework, it will be $\mathrm{d}$ for the profile who own the photo to discover that there is another profile on Facebook trying to post the same photo in the middle of hundreds of millions of photos that are being posted every day.

5. The owner of the photo will have the right to give the permission to allow the photo to be re-posted again or not on Facebook, and at the same time the watermark will protect the copyright of the photo.

\section{REFERENCES}

[1] The Top 20 Valuable Facebook Statistics - Updated January 2017, https://zephoria.com/top-15-valuablefacebook-statistics/ Access at 26/1/2017.

[2] The 2016 State of Social Marketing Report, http://simplymeasured.com/blog/introducing-the-2016state-of-social-marketingreport/\#sm.0000ug62bkrpxe4b10tygm5aqq5vr Access at 25/1/2017.

[3] Zhu Yuefeng, Lin Li, "Digital Image Watermarking Algorithms Based on Dual Transformation Domain and Self-Recovery", International Journal on Smart Sensing and Intelligent Systems Vol. 8, NO. 1, March 2015.

[4] Mr. Krunal R patel Mr .Lokesh P. Gagnani, "Current classification and introduction of Watermarking Techniques in Digital Images", International Journal of Engineering Research and Applications (IJERA) ISSN: 2248-9622 www.ijera.com Vol. 3, Issue 1, January February 2013, pp.840-846.

[5] St'ephane Bounkong, Bor'emi Toch, David Saad, David Lowe, "ICA for Watermarking Digital Images", Journal of Machine Learning Research 4 (2003) 1471-1498.

[6] Facebook Help Center, Policies and Reporting, Copyright, https://www.facebook.com/help/1020633957973118?hel pref=hc_global_nav Access at 15/1/2017.

[7] Facebook Desktop Help, Copyright Report Form, https://www.facebook.com/help/contact/1409697672616 547 Access at 15/1/2017.

[8] Facebook Help Center, Reporting Copyright Infringements, https://www.facebook.com/help/400287850027717/ Access at 16/1/2017.

[9] Jason Hiney, Tejas Dakve, Krzysztof Szczypiorski, Kris Gaj, "Using Facebook for Image Steganography", https://pdfs.semanticscholar.org/483b/0a656ef5fded842e c6dc17dd21aeec32bb66.pdf Access at 5/1/2017. 